\title{
NOVEL ALGORITHM FOR COLOR IMAGE DEMOSAIKCING USING LAPLACIAN MASK
}

\author{
Nivedita Chatterjee ${ }^{1}$, Avinash Dhole ${ }^{2}$ \\ ${ }^{I}$ M. Tech Scholar, Dept. of CSE, Raipur Institute of Technology, Raipur, C.G., India \\ ${ }^{2}$ HOD, Dept. of CSE, Raipur Institute of Technology, Raipur, C.G., India
}

\begin{abstract}
Images in any digital camera is formed with the help of a monochrome sensor, which can be either a charge-coupled device(CCD) or complementary metal oxide semi-conductor(CMOS). Interpolation is the base for any demsoaicking process. The input for interpolation is the output of the Bayer Color Filter Array which is a mosaic like lattice structure. Bayer Color Filter Array samples the channel information of $R, G$ and $B$ values separately assigning only one channel component per pixel. To generate a complete color image, three channel values are required. In order to find those missing samples we use interpolation. It is a technique of estimating the missing values from the discrete observed samples scattered over the space. Thus Demosaicking or De-bayering is an algorithm of finding missing values from the mosaic patterned output of the Bayer CFA. Interpolation algorithm results in few artifacts such as zippering effect in the edges. This paper introduces an algorithm for demosaicking which outperforms the existing demosaciking algorithms. The main aim of this algorithm is to accurately estimate the Green component. The standard mechanism to compare the performance is PSNR(Peak Signal to Noise Ratio) and the image dataset for comparison was Kodak image dataset. The algorithm was implemented using Matlab2009B version.
\end{abstract}

Keywords: Demosaicking, Interpolation, Bayer CFA, Laplacian Mask, Correlation.

\section{INTRODUCTION}

In cameras, there resides a sensor which is used to capture the image information. Using these sensors resulted in contributing $15-25 \%$ of the price of the camera. In order to reduce the price of cameras, Color Filter Arrays were used. For Demosaicking we use Bayer Color Filter Array[13]. This is the best known CFA which replaced the monochromatic sensors which was used separately for Red, Green and Blue channels resulting in three sensors. Thus Bayer CFA can be assumed as a replacement to the sensors[12].

The typical lattice arrangement of the Bayer pattern makes it possible for being the largely used CFA. The arrangement of this filter is shown in Fig.1. It may be observed that only one color value is assigned out of R,G and B channel per pixel. For any $\mathrm{NxN}$ filter there exists $50 \%$ of green component and $25 \%-25 \%$ of the red and blue components[1]. Bayer CFA separates the color components and arranges them in the specified pattern of alternate arrangement with the green components. This is a mosaic pattern of incomplete color samples, as for any color image there is $\mathrm{R}, \mathrm{G}$ and $\mathrm{B}$ components. To find those missing color, interpolation is used. Hence termed as Demosaicking, where missing color components are calculated from the sampled values. Thus demosaicking helps in recontruction of a full color image from incomplete color samples. Due to interpolation, the newly reconstructed image suffers from artifacts like zippering effects or aliasing effect[2][16]. These artifacts are the errors which do not appear in the original image. Demosaciking methods can be divided into two major categories- one being the interpolation on channels separately and the latter being the inter-channel correlation. Inter channel correlation gives better results as compared to interpolation[4],[5].

\begin{tabular}{|l|l|l|l|l|}
\hline R1 & G2 & R3 & G4 & R5 \\
\hline G6 & B7 & G8 & B9 & G10 \\
\hline R11 & G12 & R13 & G14 & R15 \\
\hline G16 & B17 & G18 & B19 & G20 \\
\hline R21 & G22 & R23 & G24 & R25 \\
\hline
\end{tabular}

Fig -1: Bayer CFA

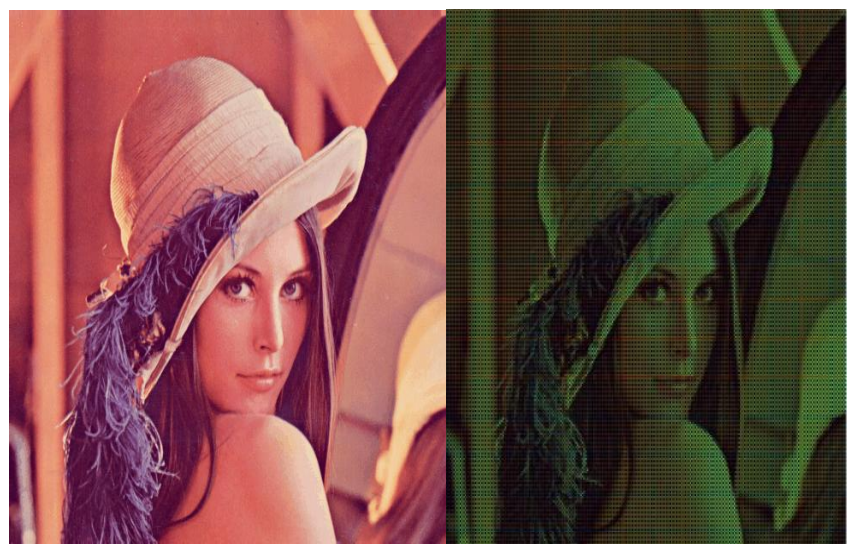

Fig -2: Original image(left) and Output of Bayer CFA(right) 
Demosaicking results in formation of artifacts which can be observed in Fig-3. This artifact results in poor quality of restored image.

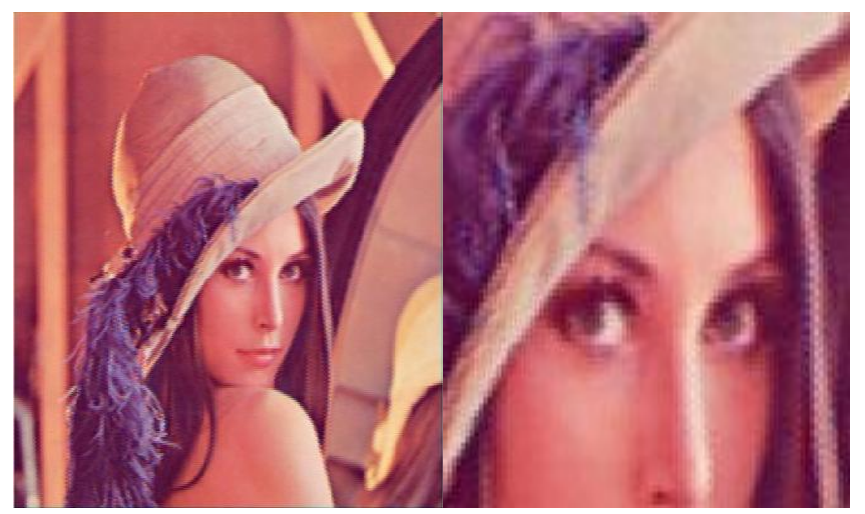

Fig -3: Interpolated image(left)and artifacts zoomed(right)

Few algorithms have been implemented which shows visible artifacts. In this paper, we have proposed a new method which results in 8-10dB improvement of the CPSNR when compared to the original image as well as the previous existing algorithms.

\section{EXISTING METHODS}

Demosaicking methods has been divided into two parts-

(i)Simple Interpolation and (ii) Correlation. In simple interpolation techniques covered are nearest neighbor interpolation, bilinear interpolation, and bicubic spline interpolation. In the first group the zippering artifact appeared at a higher ratio. In the correlation category, edge directed interpolation and smooth hue transition are placed. Interchannel correlation resulted in better images[3]. Gunturk et.al proposed a method with a combined approach of bilinear interpolation applied to red and blue channels and edge directed interpolation applied for green channel separately [3]. Another algorithm proposed by Kimmel used an iterative scheme where edge directed interpolation was combined with smooth hue transition[5]. The main steps of this algorithm were- (i) interpolate green channel, (ii) compute red and blue values using using this green information. A new algorithm was proposed, which was same as the above algorithm, after interpolation a third step was added that was the correction stage[6]. It was a high quality algorithm which eliminated the zippering effects. Few algorithm exists which has a high degree of complexity for the green channel interpolation especially. A combination of Kimmel algorithm and Optical Recovery resulted in better image restoration due to high complexity of the green channel, named as Aqua-2 algorithm If the color direction vector coincides with the gray color axis, in that case Alternating Projection method works well. All the advantages of these methods were combined altogether and when implemented produced better results[6]. Table-I shows the PSNR comparisons measured in $\mathrm{dB}$.

There exists high correlation between R,G and B channels, therefore color correlation was preferred for Demosaicking. Due to this cross correlation between the channels was calculated and range was from 0.25 to 0.99 , having average values 0.86 for red/green, 0.92 for green/blue and 0.79 for red/blue[7]. A model suggested by J.E Adams. Jr[8], two new constants were introduced $K_{B}$ and $K_{R}$. They can be calculated by $\mathrm{K}_{\mathrm{B}}=\mathrm{G}-\mathrm{B}$ and $\mathrm{K}_{\mathrm{R}}=\mathrm{G}-\mathrm{R}$. Instead of calculating the values domain-wise, it was transformed into the terms of new constants. The results outperformed with improvement in the green channel of $6.34 \mathrm{~dB}$ over the bilinear method and an average of $7.69 \mathrm{~dB}$ development on the R,G and B channels[7].

Table -1: PSNR comparison of above mentioned algorithms

\begin{tabular}{|l|l|}
\hline Method & PSNR \\
\hline Bilinear & 27.5 \\
\hline Kimmel & 33.5 \\
\hline Aqua-2 & 34.63 \\
\hline Alternating projections & 35.24 \\
\hline High-quality algorithm & 37.1 \\
\hline
\end{tabular}

As suggested by Freeman, the algorithm was Median-based interpolation comprising of two steps. First step consisted of linear interpolation and second step was using a median filter of $3 \times 3$ window[9]. Another algorithm suggested by Laroche et. al, used a gradient based concept which has calculated the color difference between the red/green and blue/green channel and then was interpolated[10]. Adaptive color plane interpolation suggested by Hamilton and Adams[11] was a modification of the gradient based interpolation where classifiers, $\alpha$ and $\beta$ were used and depending on the value of these classifiers suitable value could be assumed for that particular channel. Lei Zhang et. al proposed a method assuming the Primary Difference signal between the green and the red/blue channels and estimating the values both in horizontal and vertical directions. There was significant improvement in the PSNR value[16]. The same author proposed an algorithm which fused the local directional interpolation and non local adaptive thresholding[17]. The algorithm outperformed the state-of-the-art demosaicking methods.

\section{METHODOLOGY}

\subsection{For Red Channel}

The input image is taken and Bayer pattern is generated. Color difference interpolation is applied for green pixel and this is the guide image. Compute tentative estimate of Horizontal Red-pixel $\left(\widetilde{R^{H}}\right)$ by applying guide filter to the guide image obtained and Bayer pattern of Red pixel image(R). Compute the residual Red-pixel image ( $\mathrm{R}-\widetilde{R^{H}}$ ) by minimizing the laplacian energy. Apply bilinear interpolation in residual domain obtained in residual-red pixel image to get the final Red channel Horizontal image. Similarly vertical red channel values is calculated. 


\subsection{For Blue Channel}

The input image is taken and converted to Bayer Pattern array. Color difference interpolation method is applied for Green Pixel and considered as guide image. Now calculate the tentative estimate of horizontal Blue-pixel $\left(\widetilde{B^{H}}\right)$ by applying guide filter to the guide image obtained and Bayer pattern of Blue pixel image(B). Compute the residual Bluepixel image $\left(\mathrm{B}-\widehat{B^{H}}\right.$ ) by minimizing the laplacian energy. Apply bilinear interpolation in residual domain obtained to get the final Blue channel Horizontal image. Similarly vertical blue pixel value is calculated.

\section{RESULTS}

Table -2: Result of Kodak Dataset

\begin{tabular}{|l|l|l|l|l|}
\hline Image & \multicolumn{4}{l}{$\begin{array}{l}\text { Proposed } \\
\text { Method }\end{array}$} \\
\hline & RED & GREEN & BLUE & CPSNR \\
\hline kodim1 & 35.9907 & 38.44812 & 36.35484 & 36.80436 \\
\hline kodim2 & 38.41384 & 43.88 & 41.83973 & 40.78352 \\
\hline kodim3 & 42.43939 & 45.81395 & 41.71374 & 42.99188 \\
\hline kodim4 & 38.20758 & 44.30077 & 42.82978 & 40.96257 \\
\hline kodim5 & 37.29591 & 39.90501 & 36.47861 & 37.66519 \\
\hline kodim6 & 38.96318 & 41.09398 & 37.92634 & 39.13763 \\
\hline kodim7 & 42.35421 & 45.28166 & 41.68563 & 42.85047 \\
\hline kodim8 & 34.09049 & 37.20012 & 34.096 & 34.9042 \\
\hline kodim9 & 41.67074 & 44.55918 & 41.51579 & 42.37561 \\
\hline kodim10 & 41.40061 & 44.94254 & 41.31518 & 42.25849 \\
\hline kodim11 & 38.39765 & 41.05125 & 38.92885 & 39.31696 \\
\hline kodim12 & 42.22411 & 46.01401 & 42.5024 & 43.27399 \\
\hline kodim13 & 33.14832 & 34.43473 & 32.16007 & 33.14954 \\
\hline kodim14 & 36.47877 & 40.40548 & 36.9419 & 37.62566 \\
\hline kodim15 & 37.12953 & 42.44894 & 40.05859 & 39.34019 \\
\hline kodim16 & 42.61606 & 44.68774 & 41.83687 & 42.88918 \\
\hline kodim17 & 41.01202 & 42.55487 & 40.02356 & 41.07533 \\
\hline kodim18 & 36.03399 & 37.91994 & 35.899 & 36.52496 \\
\hline kodim19 & 39.2145 & 41.75231 & 39.25909 & 39.92499 \\
\hline kodim20 & 41.48291 & 43.16091 & 38.63451 & 40.68346 \\
\hline kodim21 & 38.1504 & 40.16923 & 36.86723 & 38.19116 \\
\hline kodim22 & 38.19407 & 40.73273 & 37.54817 & 38.62325 \\
\hline kodim23 & 42.63697 & 46.25818 & 43.34106 & 43.81984 \\
\hline kodim24 & 35.36084 & 36.85233 & 32.81754 & 34.68463 \\
\hline
\end{tabular}

Table- 2 shows the results of the proposed algorithm when applied to 24 images of Kodak Dataset [13]. Table -3 shows the results when the same algorithm was applied to the 18 images of IMAX database (McMaster Database) [15]. Table- 4 shows the comparison of the Previous algorithms with the proposed algorithm on IMAX Dataset.
Table -3: Result of IMAX Dataset

\begin{tabular}{|l|l|l|l|l|}
\hline \multicolumn{5}{|l}{$\begin{array}{l}\text { Proposed } \\
\text { Method }\end{array}$} \\
\hline Image & RED & GREEN & BLUE & CPSNR \\
\hline IMAX1 & 29.21229 & 32.42841 & 26.9258 & 28.97315 \\
\hline IMAX2 & 34.66927 & 39.26991 & 33.3025 & 35.1003 \\
\hline IMAX3 & 34.17191 & 36.78554 & 31.96751 & 33.87931 \\
\hline IMAX4 & 38.30484 & 41.34507 & 35.35203 & 37.67242 \\
\hline IMAX5 & 36.89551 & 37.86462 & 30.85081 & 34.01581 \\
\hline IMAX6 & 39.05702 & 41.83149 & 35.93489 & 38.28932 \\
\hline IMAX7 & 37.542 & 39.38773 & 36.1445 & 37.49397 \\
\hline IMAX8 & 34.14455 & 41.68364 & 38.24871 & 36.97088 \\
\hline IMAX9 & 34.20242 & 41.32974 & 36.50707 & 36.46461 \\
\hline IMAX10 & 37.62951 & 42.0721 & 37.59404 & 38.65739 \\
\hline IMAX11 & 39.0232 & 41.9884 & 39.38727 & 39.94764 \\
\hline IMAX12 & 40.25507 & 42.15243 & 37.75307 & 39.67949 \\
\hline IMAX13 & 42.23337 & 44.91082 & 37.64852 & 40.55644 \\
\hline IMAX14 & 39.33683 & 42.85624 & 36.42193 & 38.79168 \\
\hline IMAX15 & 36.91814 & 42.46938 & 39.09144 & 38.93668 \\
\hline IMAX16 & 34.38471 & 35.24319 & 35.74638 & 35.08797 \\
\hline IMAX17 & 31.25378 & 36.96613 & 31.52874 & 32.58689 \\
\hline IMAX18 & 34.99269 & 37.61057 & 36.16048 & 36.12495 \\
\hline
\end{tabular}

Table -4: Comparison of IMAX Dataset with other algorithms

\begin{tabular}{|l|l|l|l|l|}
\hline & \multicolumn{3}{|c|}{ PSNR } & \multirow{2}{*}{ CPSNR } \\
\cline { 1 - 4 } Algorithms & Red & Green & Blue & \multirow{2}{*}{ CPS.49 } \\
\hline Hirakawa & 33.00 & 36.98 & 32.16 & 34.47 \\
\hline LMMSE & 34.03 & 37.99 & 33.04 & 35.20 \\
\hline NAT & 36.28 & 39.76 & 34.39 & 36.62 \\
\hline Proposed & 36.34 & 39.89 & 35.36 & \\
\hline
\end{tabular}

The proposed algorithm results in better images of the Imax dataset as shown in Table-4 and as shown in Chart 1, the proposed method results in significant improvement in the PSNR as compared to the bilinear method,which is a basic method. The evaluation of algorithm was done on the basis of Mean Square Error(MSE) whereas Peak Signal to Noise Ratio(PSNR) can be calculated as $\mathrm{PSNR}=10 \log _{10}$ [255^2/MSE]. Table-5 also shows the comparison of the results as presented by S.C Pei et.al[7]. Comparing the PSNR channel wise on the specified image, the proposed method performed well with a significant improvemnt 7$8 \mathrm{~dB}$ in the PSNR values. 


\section{CONCLUSION}

In this paper we proposed a novel method for color image demosaicking which can be one of the alternative to the various currently used algorithms. Using a laplacian mask helps to minimize the error formation in the demosaicked image. Experimental results show that the proposed algorithm outperforms the various above mentioned algorithms on the Kodak as well as IMAX dataset.

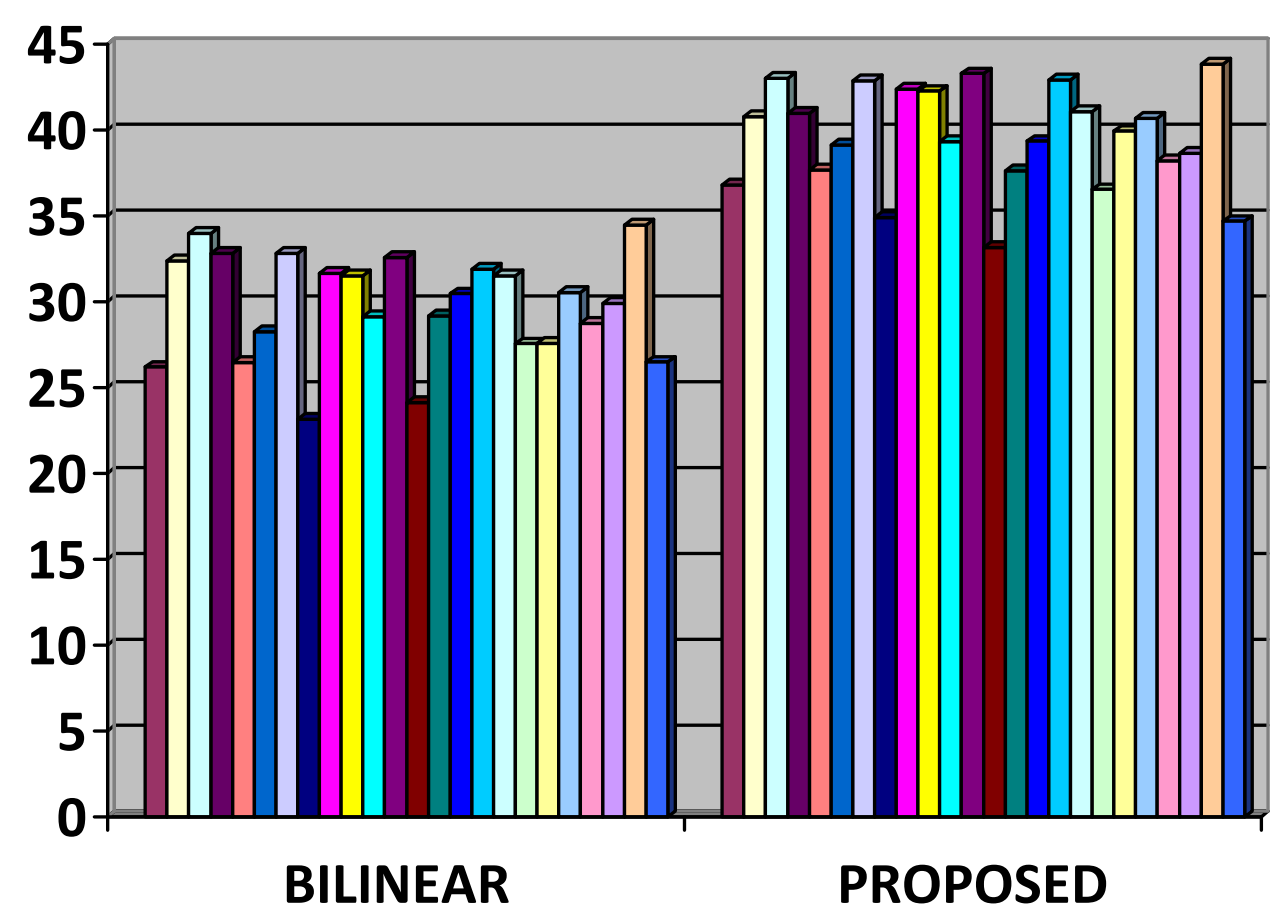

Chart -1: Results of 24 Kodak Images compared to Bilinear

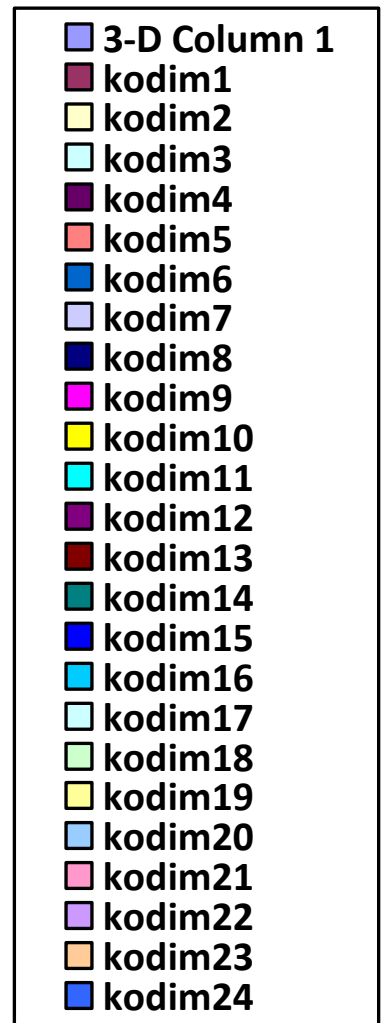

Table -5: Comparison of Kodak Dataset with other algorithms

\begin{tabular}{|l|l|l|l|l|l|l|l|l|l|}
\hline \multirow{2}{*}{ Image } & \multicolumn{1}{|l|}{ E } & \multicolumn{1}{l}{ M } & \multicolumn{1}{l|}{ S } & \multicolumn{1}{l|}{ M } & \multicolumn{2}{l|}{ PROPOSED } \\
\cline { 2 - 11 } & $\mathrm{r}$ & $\mathrm{g}$ & $\mathrm{b}$ & $\mathrm{r}$ & $\mathrm{g}$ & $\mathrm{b}$ & $\mathrm{r}$ & $\mathrm{g}$ & $\mathrm{b}$ \\
\hline Cap & 30.8 & 35.5 & 31.3 & 35.7 & 41.2 & 35.0 & 42.4 & 45.8 & 41.7 \\
\hline Motor & 22.6 & 27.5 & 24.2 & 30.1 & 34.7 & 29.7 & 37.2 & 39.9 & 36.4 \\
\hline Airplane & 29.5 & 32.9 & 28.2 & 33.8 & 38.4 & 32.6 & 41.4 & 43.1 & 38.6 \\
\hline Parrot & 30.9 & 36.3 & 32.9 & 35.8 & 41.9 & 36.6 & 42.6 & 46.2 & 43.3 \\
\hline
\end{tabular}

\section{REFERENCES}

[1]. Ramanath, R. , Snyder, W. E. , Bilbro, G. L. , and Sander III, W. A. , "Demosaicking methods for Bayer color arrays", Journal of Electronic Imaging, Vol.11( 3), pp. 306-315,July 2002.

[2]. Hirakawa, K. ,Parks, T.W. , "Adaptive homogeneitydirected demosaicing algorithm", IEEE Transactions on Image Processing, Vol.14(3), pp. 360-369, 2005.

[3]. Gunturk, B. K. , Altunbasak, Y. , and Mersereau, R. , "Color plane interpolation using alternating projections", IEEE Transactions on Image Processing, Vol. 11(9), pp.997-1013, Sept. 2002.
[4]. T. Kuno, and H. Sugiura "New Interpolation Method Using Discriminated Color Correlation for Digital Still Cameras”, IEEE Trans. Consumer Electronic, Vol.45(1), pp. 259-267 ,Feb. 1999

[5]. R. Kimmel, "Demosaicing: Image Reconstruction from Color CCD Samples", IEEE Trans. Image Processing, Vol. 8(9), pp. 1221-1228, Sep. 1999.

[6]. Lukin, A. , Kubasov, D. , "High-Quality Algorithm for Bayer Pattern Interpolation", Programming and Computer Software, Vol. 30(6), pp. 347-358, 2004.

[7]. Pei, S.C. , and Tam, I.K. , "Effective color interpolation in CCD color filter arrays using signal correlation", IEEE Transactions on Circuits System. Video Technology, Vol 13(6), pp. 503-513, June 2003. 
[8]. Adams, Jr. J. E. , "Design of Practical Color Filter Array Interpolation Algorithms for Digital Cameras", Proceeding of SPIE, Vol. 3028, pp. 117- 125, 1997.

[9]. Freeman, W.T. , "Median filter for reconstructing missing color samples", U. S. Patent No. 4,724,395(1988).

[10]. Laroche, C. A. , and Presscott, M. A. , "Apparatus and method for adaptively interpolating a full Color image utilizing chrominance gradients", U.S.Patent No. 5,373,322 (1994).

[11]. Hamilton, J. F. , an Adams, J. E. , "Adaptive color plane interpolation in single sensor color electronic camera" ,U.S. Patent No. 5,629,734(1997).

[12]. Adams, Jr J.E. , "Interactions between Color Plane Interpolation and Other Image Processing Functions in Electronic Photography" ,Proceeding of SPIE, Vol. 2416, pp. 144-151, 1995.

[13].Kodak Lossless True Color Image Suite[EB/OL]. http: //rok.us.graphics/kodak/,1999.

[14].B.E Bayer, "Color imaging array," U.S. Patent No. 3,971,065(1976).

[15].http://www4.comp.polyu.edu.hk/ cslzhang/CDM_Data set.htm

[16]. Zhang, L. and Wu, X., "Color demosaicking via directional linear minimum mean square-error estimation," Image Processing, IEEE Transactions on 14(12),21672178(2005).

[17]. Zhang, L., Wu, X., Buades, A., and Li, X., "Color demosaicking by local directional interpolation and nonlocal adaptive thresholding," Journal of Electronic Imaging 20(2), 023016-023016 (2011).

\section{BIOGRAPHIES}

Nivedita Chatterjee ${ }^{1}$ is a P. G Student (M. Tech) in the Department of Computer Science and Engineering, Raipur Institute of Technology, Raipur(C.G). She received her Bachelor of Engineering (CSE) in 2010 from Raipur Institute of Technology, Raipur which is affilated to Chhattisgarh Swami Vivekanand Technical University, Bhilai (C.G). Her research interest are Digital Image Processing, Computer networks, ANFIS and Neural Networks

Avinash Dhole is an Avinash Dhole ${ }^{2}$ is an Associate Professor and Head in Computer Science and Engineering Department, in Raipur Institute Of Technology, Raipur, (C.G.) . His research interests include Digital Image Processing, Compilers, Automata Theory, Neural Network, Artificial Intelligence, Information and Network Security 